\title{
HOXA9 inhibitors promote microcirculation of coronary arteries in rats via downregulating E-selectin/VCAM-1
}

\author{
SHILEI LIU, JING GAO and SAI WANG
}

Internal Medicine-Cardiovascular Department, Shengli Oilfield Central Hospital, Dongying, Shandong 257000, P.R. China

Received September 1, 2020; Accepted April 28, 2021

DOI: $10.3892 /$ etm.2021.10303

\begin{abstract}
Atherosclerosis (AS) is a chronic pathophysiological process that causes high mortality and morbidity. It has previously been reported that homeobox A9 (HOXA9) may participate in regulation of the cardiovascular system and the pathology of AS by upregulating E-selectin and vascular cell adhesion molecule-1 (VCAM-1). Thus, inhibiting HOXA9 could promote microcirculation of coronary arteries and could act as a potential therapy for AS treatment. Sprague-Dawley rats were randomly divided into four groups, as follows: i) AS; ii) AS + HOXA9 inhibitor; iii) AS + small interfering RNA-HOXA9 and iv) normal control. ELISA was used to measure the levels of TNF- $\alpha$, IL-1 $\beta$, IL-12, C-C motif chemokine ligand 25 (CCL25), low-density lipoprotein (LDL), high-density lipoprotein (HDL) and very-low-density lipoprotein (VLDL). Flow cytometry was employed to detect the content of M1 macrophages. Hematoxylin \& eosin (H\&E) staining was performed to observe the morphology of the coronary arteries. Oil red O staining was conducted for the evaluation of lipid accumulation. Immunohistochemistry was used to visualize the protein expression levels of HOXA9 in the coronary arteries. Western blotting was utilized to determine the protein expression levels of HOXA9, platelet factor-4 (PF4), E-selectin and VCAM-1. HOXA9 inhibitors were found to downregulate the levels of TNF- $\alpha$, IL-1 $\beta$, IL-12, CCL25, LDL and VLDL, and upregulate HDL levels in the blood of AS rats. The content of M1 macrophages was also decreased following injection of HOXA9 inhibitors in the AS group. H\&E and oil red $\mathrm{O}$ staining analysis indicated that HOXA9 inhibitors attenuated vascular symptoms and lipid formation in AS rats. Furthermore, western blotting suggested that inhibition of HOXA9 reduced the expression levels of PF4, E-selectin and VCAM-1, while overexpression of PF4 resulted in the opposite effects. The present study revealed that inhibiting HOXA9
\end{abstract}

Correspondence to: Dr Sai Wang, Internal MedicineCardiovascular Department, Shengli Oilfield Central Hospital, 31 Jinan Road, Dongying, Shandong 257000, P.R. China

E-mail:ws200209@aliyun.com

Key words: atherosclerosis, coronary arteries, homeobox A9 inhibitors, vascular cell adhesion molecule-1 alleviated the symptoms of AS via downregulation of the PF4 and E-selectin/VCAM-1 pathway to promote microcirculation in the coronary arteries of AS rats. These findings indicated that HOXA9 inhibitors may have the potential to succeed in the treatment of AS.

\section{Introduction}

Atherosclerosis (AS), a cardiovascular disease with complex interactions, is regarded as one of the leading causes of morbidity and mortality worldwide (1). AS is a chronic pathophysiological process that is initially inapparent until intimal thickening of coronary arteries diminishes blood flow. This is accompanied by inflammatory phenomena, such as accumulation of lipids, aggravation of inflammatory lesions, thrombosis and fibrous hyperplasia (2). The thickening of the artery intima can be characterized by the deposition of low-density lipoproteins (LDLs), and adhesion of necrotic cellular debris and immune cells, which facilitates the formation of atherosclerotic plaques $(3,4)$. In the early stages of AS, endothelial cells (ECs) and vascular smooth muscle cells (VSMCs) are activated, inducing the expression of inflammatory mediators and adhesive factors; these include E-selectin, P-selectin, vascular cell adhesion molecule-1 (VCAM-1) and the glycoprotein intercellular adhesion molecule-1 (ICAM-1) $(5,6)$. The migration and recruitment of platelets and inflammatory monocytes are stimulated by $\mathrm{C}-\mathrm{C}$ motif chemokine ligands (CCL), including CCL2, CCL3 and CCL25. Following on from this, the monocytes mature into M1 and M2 macrophages, and secrete inflammatory cytokines, such as TNF- $\alpha$, IL-1 $\beta$ and IL-12, which maintain inflammation and promote chemotaxis of immune cells toward the plaque. As a consequence of abnormal absorption of modified LDLs, macrophages develop further into foam cells, a process induced by platelet-released platelet factor-4 (PF4) or stromal cell-derived factor-1 (7-9). Therefore, the identification of expression levels of these inflammatory mediators and chemokines has the potential to improve the understanding of pathophysiological processes of $\mathrm{AS}$, and the regulation of related signaling pathways may contribute to the treatment of AS (10). Homeobox (HOX) genes are transcription factors that act during normal development (11) and can be categorized into four groups based on the similarity of a 61-amino acid homeodomain, namely: i) HOXA; ii) HOXB; iii) HOXC and iv) HOXD (12). Several studies have demonstrated that HOX genes may participate in the regulation 
of the cardiovascular system and the pathology of AS (13). Among them, HOXA9 was found to be involved in regulating cellular processes of ECs and HOXA9 inhibition was revealed to suppress E-selectin expression in response to inflammatory cytokines (14). Therefore, in the present study, HOXA9 inhibition was hypothesized to promote microcirculation of coronary arteries through downregulating PF4, E-selectin and VCAM-1, thus revealing the pro-inflammatory role of HOXA9 in AS rats.

\section{Materials and methods}

Modeling of AS. AS was established in Sprague Dawley (SD) rats were intravenously injected with vitamin D3 (Sigma-Aldrich; Merck KGaA) at a dose of 500,000 IU/kg/d for 3 days, and then orally fed with a high-fat and high-cholesterol diet for 3 months. Rats were housed in an environmentally controlled room (50-60\% humidity, 20-25 C) with $12 \mathrm{~h}$ light/dark cycle and with free access to food and water. The detailed feeds were purchased from ReadyDietech Co., Ltd, according to a previous study (15). Following the intravenous vitamin D3 injection, the gross anatomy and histology of the model rats were constantly monitored. Successful model establishment was indicated by the occurrence of vascular intimal injury and inflammation induced by mechanical effects, such as hypercalcemia or balloon aortic laceration.

Tested animals grouping. Male SD rats (weight, 180-200 g; age, 4-6 weeks) were purchased from Shanghai SLAC Laboratory Animal Center. A total of 56 rats were randomly divided into four experimental groups $(n=14)$; i) AS+HOXA9-inhibitor group: AS model rats intravenously injected with HOXA9 inhibitors (anti-HOXA9 antibody, cat. no. SAB2108152; Sigma-Aldrich; Merck KGaA) at a dose of $100 \mu \mathrm{g} / \mathrm{kg}$ body weight; ii) AS+si-HOXA9 group: AS model rats intravenously injected with HOXA9 small interfering (si)RNA; iii) AS group: AS model rats were intravenously injected with $100 \mu \mathrm{g} / \mathrm{kg}$ saline; iv) $\mathrm{NC}$ group: Control rats were housed under the same conditions but fed with a normal diet. Rats were intravenously injected with saline $(100 \mu \mathrm{g} / \mathrm{kg})$. For construction of the overexpression models of PF4, E-selectin and VCAM-1, the overexpression vector of each gene was constructed by HanBio Biotechnology Co., Ltd., via a lentivirus expressing system. The lent-OE-PF4, lent-OE-E-selectin and lent-OE-VCAM-1 vectors were separately injected into rats in the AS+HOXA9-inhibitor group $(n=3$ for each vector) via tail vein injection $\left(1.0 \times 10^{9} \mathrm{TU} / \mathrm{ml}, 10 \mu \mathrm{l}\right)$, whereas lent-OE-negative control (the coding region was substituted by GFP) was used as a control. All the tested animals were housed based on the standard of the National Institutes of Health Guide for the Care and Use of Laboratory Animals (NIH Publication). Following the aforementioned treatment for 3 months, all tested animals were intraperitoneally anesthetized with pentobarbital $(60 \mathrm{mg} / \mathrm{kg})$ and then sacrificed via cervical dislocation. Blood samples were collected from the abdominal artery for subsequent analysis. The coronary arteries and thoracic aorta samples were harvested and stored in a frozen state using liquid nitrogen.

ELISA for inflammatory cytokines. The collected blood samples were placed at room temperature for 10-20 min to coagulate naturally, followed by centrifugation for $20 \mathrm{~min}$ $\left(1,000 \mathrm{x} \mathrm{g}, 4^{\circ} \mathrm{C}\right)$. The supernatant was collected, and centrifugation was repeated once under the same conditions in case of precipitation during storage. Following the above sample pre-treatment, ELISA kits (Nanjing Jiancheng Bioengineering Institute) were utilized to detect the levels of inflammatory cytokines, TNF- $\alpha$ (cat. no. H052-1), IL-1 $\beta$ (cat. no. H002), IL-12 (cat. no. H010) and CCL25 (cat. no. H458-1), as well as LDL (cat. no. A113-1-1), very-LDL (VLDL; cat. no. H249) and high-density lipoprotein (HDL; cat. no. A112-1-1) levels, according to the manufacturer's protocols.

Flow cytometric analysis of M1 macrophages. The blood samples were pre-treated with $1 \%$ heparin(Sigma-Aldrich;Merck $\mathrm{KGaA}$ ) for anticoagulation within $30 \mathrm{~min}$ and then centrifuged at $4^{\circ} \mathrm{C}$ for $30 \mathrm{~min}(1,000 \mathrm{x} \mathrm{g})$. The detection of M1 macrophages was performed using MojoSort ${ }^{\mathrm{TM}}$ human pan monocyte isolation kit (BioLegend, Inc.) according to the manufacturer's instructions. Blood samples were diluted with PBS and gently placed on the separating liquid. After stratification, the second layer was extracted and washed with PBS to obtain mononuclear cells. The collected cells were then resuspended in a combined medium (1640 medium containing 10\% fetal bovine serum (Sigma-Aldrich; Merck KGaA), added to GM-CSF cytokines (MojoSort $^{\mathrm{TM}}$ ) to a final concentration of $10 \mathrm{ng} / \mathrm{ml}$ ) and cultured at $37^{\circ} \mathrm{C}$ in an atmosphere containing $5 \% \mathrm{CO}_{2}$ for 7 days. The M1 macrophages were labeled with CD206-A-phycoerythrin (cat.no. SAB4700690; 1:500) and CD163-A-fluorescein isothiocyanate (cat. no. F3668; 1:500) (Sigma-Aldrich; Merck KGaA) and incubated at $4^{\circ} \mathrm{C}$ for $30 \mathrm{~min}$. After incubation, the cells were washed twice with PBS and resuspended in $300 \mathrm{ml}$ PBS. The phenotypes of cells were detected using a BD FACSVerse ${ }^{\mathrm{TM}}$ Flow Cytometer (BD Biosciences) and analyzed using Cell Quest Pro 5.1 (BD Biosciences) (16).

Histological evaluation and lipid accumulation. The histological evaluation was performed for each group based on a previously published method using hematoxylin \& eosin (H\&E) staining (17). The coronary arteries and thoracic aorta samples were fixed in $10 \%$ formalin for $12 \mathrm{~h}$ at $4^{\circ} \mathrm{C}$, and then embedded in paraffin for subsequent sectioning. The $5-\mu \mathrm{m}$ specimens were stained with H\&E for 5 min at room temperature for histological evaluation, and then stained with oil red O for $10 \mathrm{~min}$ at room temperature for lipid accumulation detection, respectively. The lesion change was observed using a light microscope (magnification, x20).

Immunohistochemistry. The protein expression levels of HOXA9 in coronary arteries and thoracic aorta samples were evaluated by immunohistochemical methods based on a previous publication (18). The sample preparation procedure was the same as aforementioned, after which 5- $\mu$ m specimens were embedded in paraffin. The sections were blocked with $3 \% \mathrm{H}_{2} \mathrm{O}_{2}$ for $10 \mathrm{~min}$ at room temperature to remove endogenous peroxidase. After further blocking with PBS containing $1.5 \%$ normal horse serum (Sigma-Aldrich; Merck KGaA) at room temperature for $45 \mathrm{~min}$, the sections were incubated with the primary antibody anti-HOXA9 (Abcam; cat. no. ab191178; $1: 1,000)$ overnight at $4^{\circ} \mathrm{C}$. This was followed by the addition of secondary HRP-conjugated rabbit anti-rat $\mathrm{IgG}$ 


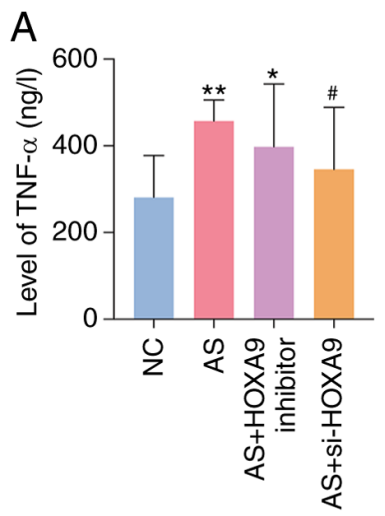

E

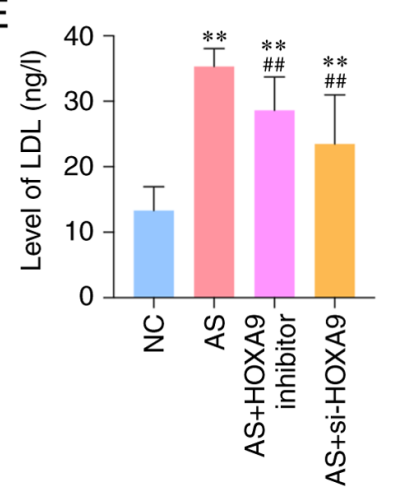

B
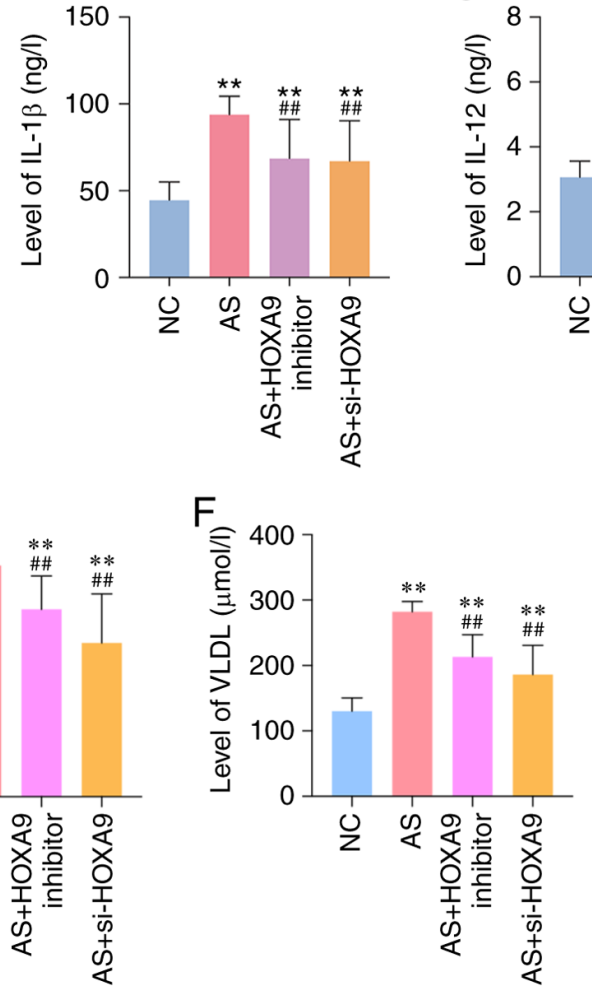

C

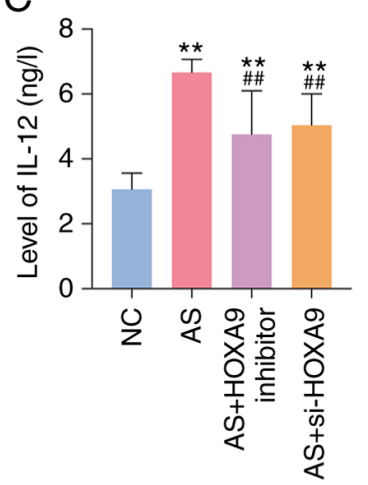

D

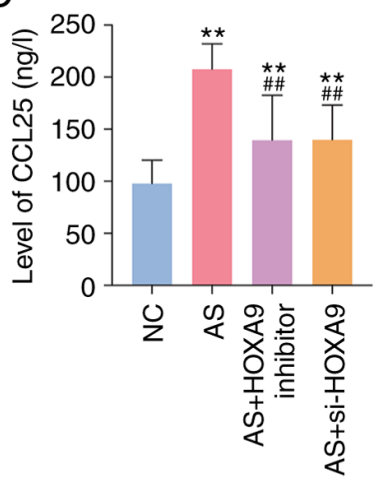

G

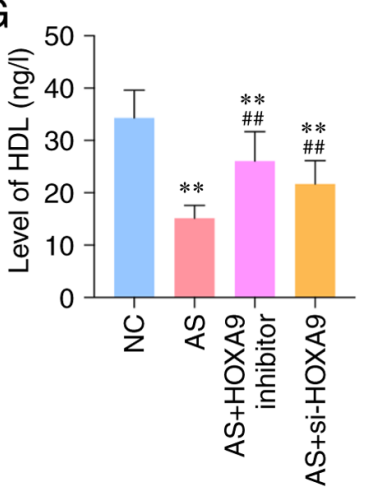

Figure 1. HOXA9 inhibitors downregulate the levels of (A) TNF- $\alpha$, (B) IL-1 $\beta$, (C) IL-12, (D) CCL25, (E) LDL, (F) VLDL and (G) HDL in blood samples of AS rats, as determined using ELISAs. ${ }^{*} \mathrm{P}<0.05,{ }^{* *} \mathrm{P}<0.01$ vs. $\mathrm{NC}$ group; ${ }^{*} \mathrm{P}<0.05,{ }^{\# \#} \mathrm{P}<0.01$ vs. AS group. AS, atherosclerosis; NC, normal control; si, small interfering; HOXA9, homeobox A9; CCL25, C-C motif chemokine ligand 25; LDL, low-density lipoprotein; HDL, high-density lipoprotein; VLDL, very-low-density lipoprotein.

(cat. no. ab6734; 1:1,000) for $30 \mathrm{~min}$ at room temperature. The scoring was conducted according to a previous study on an Olympus GX51 light microscope. The score was determined according to positive staining percentage (PSP) and calculated as follows: 0, PSP $<5 \%$; $1, \mathrm{PSP}<5-25 \% ; 2, \mathrm{PSP}<25-50 \% ; 3$, $\mathrm{PSP}<50-75 \% ; 4, \mathrm{PSP}>75 \%$ (18).

Western blotting. The coronary arteries and thoracic aorta samples were primarily homogenized on ice using RIPA buffer (Sigma-Aldrich; Merck KGaA). After being completely lysed, the sample homogenate was centrifuged at $12,000 \mathrm{x} \mathrm{g}$ for $15 \mathrm{~min}$ at $4^{\circ} \mathrm{C}$. The supernatant was preserved for protein quantification using a BCA kit and $15 \mu \mathrm{g}$ protein/lane was separated by polyacrylamide gel electrophoresis. The separated proteins were subsequently transferred onto polyvinylidene difluoride membranes at $25 \mathrm{~V}$ for $30 \mathrm{~min}$ and blocked in 5\% non-fat milk for $50 \mathrm{~min}$ at room temperature. The membranes were incubated with primary antibodies overnight at $4^{\circ} \mathrm{C}$. The primary antibodies obtained from Abcam were as follows: Anti-HOXA9 (cat. no. ab191178; 1:1,000), anti-PF4 (cat. no. ab129090; 1:1,000), anti-E-selectin (cat. no. ab18981; 1:1,000), anti-VCAM-1 (cat. no. ab134047; $1: 1,000)$ and anti- $\beta$-actin (cat. no. ab8226; 1:1,000). After washing with TBS-Tween $(0.1 \%)$ in triplicate, the membranes were incubated with goat anti-rat IgG secondary antibody (Abcam; cat. no. ab150165; 1:5,000) for $1 \mathrm{~h}$ at $37^{\circ} \mathrm{C}$. Protein bands were detected using enhanced chemiluminescent reagent (Santa Cruz Biotechnology, Inc.). The density of blots for proteins were normalized to $\beta$-actin using ImageJ 2.0 software (National Institute of Health).
Statistical analysis. The experiments were performed in triplicate and all statistical data are expressed as the mean \pm standard deviation. Comparisons between groups were made for data analyses using GraphPad Prism 7.0 (GraphPad Software, Inc.). Comparisons between multiple groups were performed by one-way ANOVA followed by Bonferroni's post hoc multiple comparisons test. $\mathrm{P}<0.05$ was considered to indicate a statistically significant difference.

\section{Results}

HOXA9 inhibitors reduce the levels of inflammatory cytokines and upregulate HDL levels in AS rats. Vascular inflammation may be an important indicator of AS modeling in rats (19). The successfully constructed inhibition or silencing models were verified using western blotting (Fig. S1). ELISAs were used to detect the levels of inflammatory cytokines in the blood of rats. As shown in Fig. 1A-C, the levels of inflammatory cytokines TNF- $\alpha$, IL-1 $\beta$ and IL-12 were significantly increased in the AS group compared with those in the normal control (NC) group. The levels of chemokine CCL25 were also significantly upregulated in the AS group compared with the NC group (Fig. 1D), which indicated the success of the AS model. The levels of LDL and VLDL were increased, and HDL was decreased in AS rats compared with the NC group, whereas inhibition or silencing of HOXA9 demonstrated opposite effects (Fig. 1E-G). The injection of HOXA9 inhibitors or si-HOXA9 markedly decreased the levels of these cytokines, as well as LDL and VLDL, in AS model rats, but were unable to completely restore them to normal levels. 
A

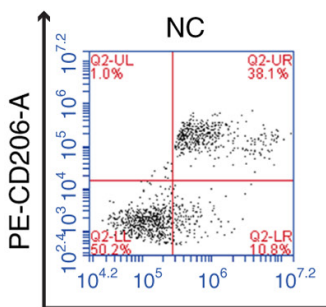

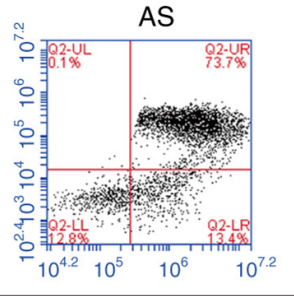

$\mathrm{B}$

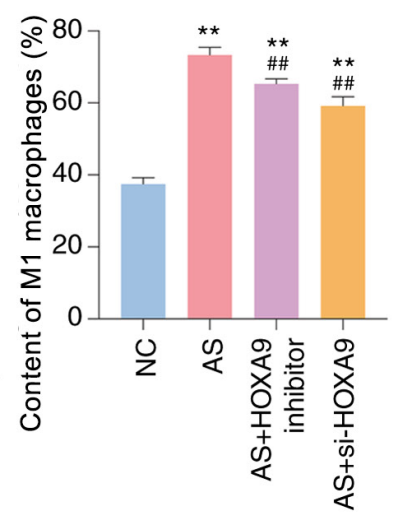

Figure 2. HOXA9 inhibitors decrease the content of M1 macrophages in blood samples of AS rats, as determined using flow cytometry. (A) Monocyte content in NC group, AS group, AS + HOXA9 inhibitor group and AS + si-HOXA9 group, as detected by flow cytometry. (B) A statistical analysis of the content of M1 macrophages. The data in Q2-UR were analyzed. ${ }^{* *} \mathrm{P}<0.01$ vs. NC group. ${ }^{\# \#} \mathrm{P}<0.01$ vs. AS group. AS, atherosclerosis; NC, normal control; si, small interfering; HOXA9, homeobox A9; PE, phycoerythrin; FITC, fluorescein isothiocyanate.

A

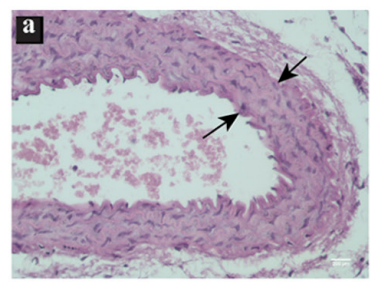

B

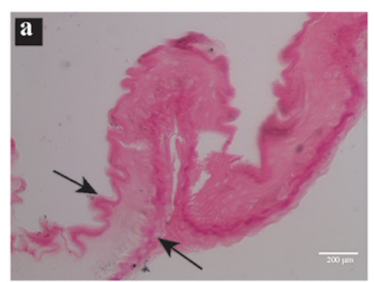

C

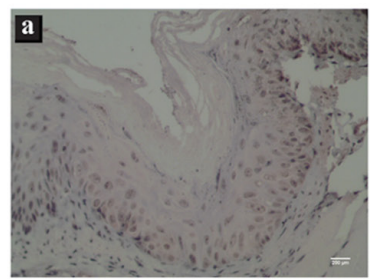

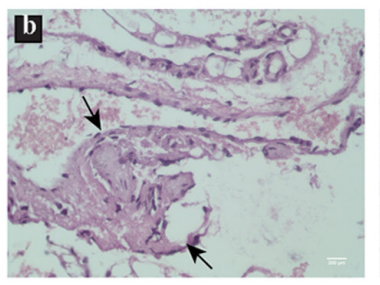
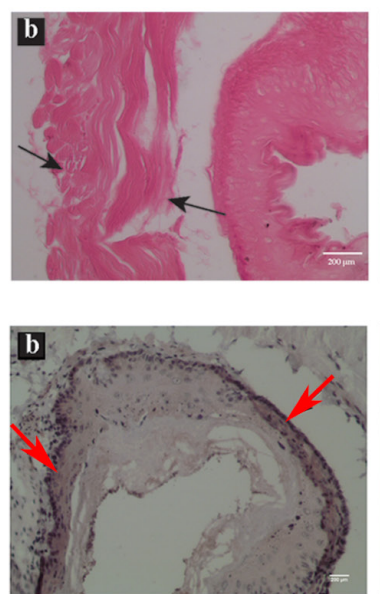
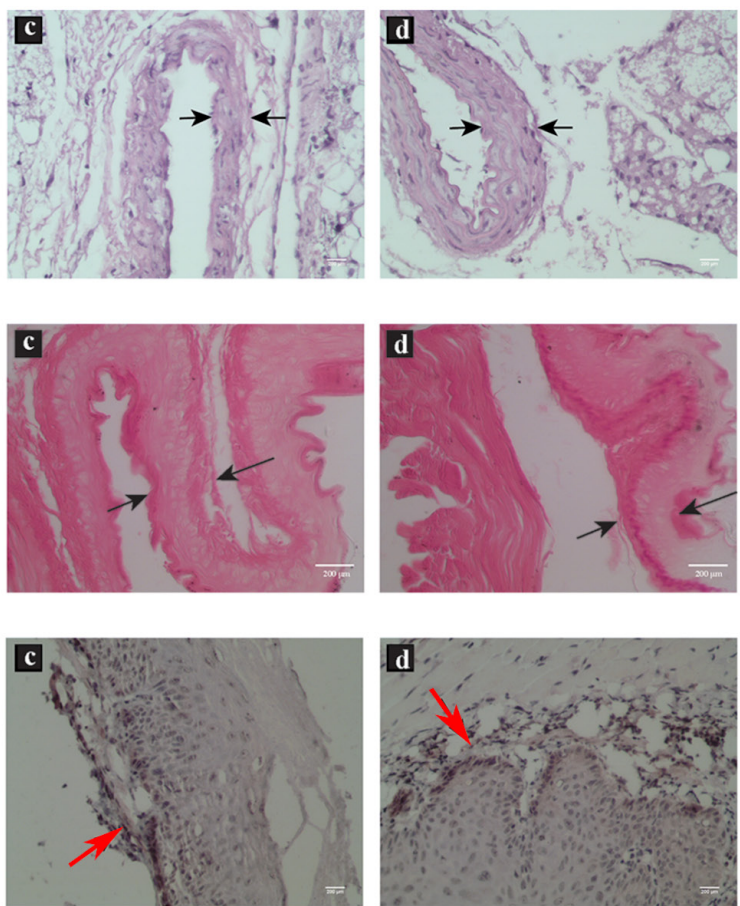

Figure 3. Effects of HOXA9 inhibitors on histology and the expression of HOXA9. HOXA9 inhibitors (A) improve coronary arteries morphology, (B) decrease lipid formation and (C) decrease the expression levels of HOXA9 of AS rats. (a) Normal control group (b) AS group (c) AS + HOXA9 inhibitor group (d) and AS + si-HOXA9 group. Black arrows refer to (A and B) the thickness of vascular wall; Red arrows (C) refer to where the expression of HOXA9 was upregulated. Scale bar, $200 \mu \mathrm{m}$. AS, atherosclerosis; si, small interfering; HOXA9, homeobox A9.

HOXA9 inhibitors decrease the content of M1 macrophages in $A S$ rats. The content of M1 macrophages may directly reflect the inflammatory status of vessels, thus acting as another indicator of AS (20). Flow cytometry revealed that the content of M1 macrophages in the AS group was $73.7 \%$, which was significantly higher than that of the NC group (Fig. 2). The observed increase was reversed when the rats were treated with a HOXA9 inhibitor or si-HOXA9. However, si-HOXA9 exhibited a larger reduction in M1 macrophage content when compared with the effects of the HOXA9 inhibitor. Taken together, these data suggested that HOXA9 inhibitors may reduce the content of M1 macrophages in AS rats.
HOXA9 inhibitors improve coronary artery morphology and inhibit lipid formation in AS rats. The coronary artery morphology of rats was observed using H\&E staining. In the $\mathrm{NC}$ group, the surface of the vascular intima was smooth and complete, and VSMCs were arranged in an orderly manner (Fig. 3A). In comparison, the vascular intima was thickened and uplifted inward, and ECs were ruptured in the AS group. After treatment with HOXA9 inhibitors or si-HOXA9, the thickness of the intima was markedly diminished and no plaque was observed compared with that in AS rats (Fig. 3A). Oil red $\mathrm{O}$ staining revealed that the inhibition of HOXA9 markedly alleviated the lipid formation compared with AS group (Fig. 3B). This evidence indicated that HOXA9 inhibitors may 

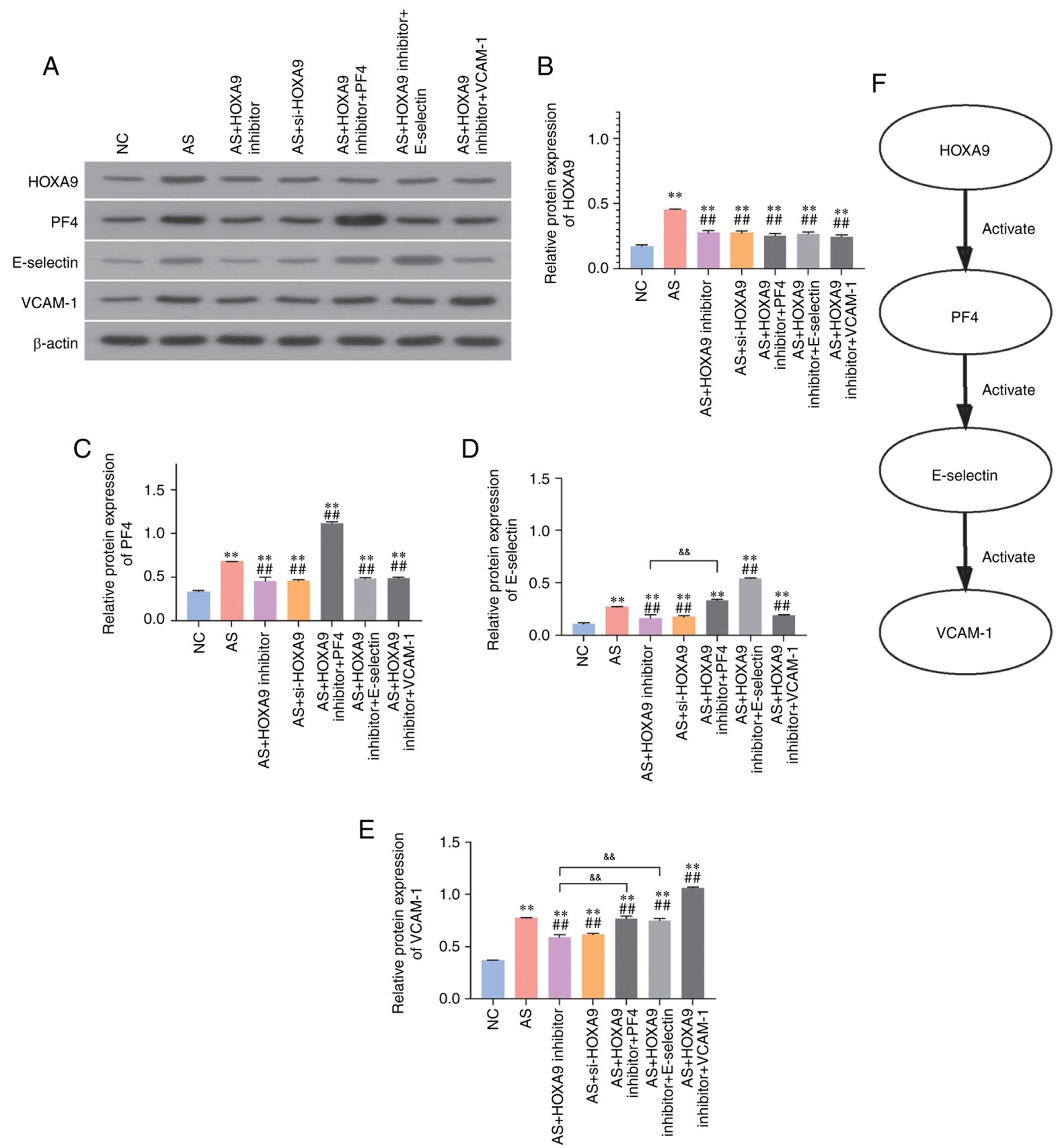

Figure 4. HOXA9 inhibitors alleviate damage induced by AS via downregulating PF4 and E-selectin/VCAM-1 proteins in coronary arteries and thoracic aorta samples. (A) Results of western blotting. Relative expression levels of (B) HOXA9, (C) PF4, (D) E-selectin and (E) VCAM-1 normalized to $\beta$-actin. (F) Association between HOXA9, PF4 and E-selectin/VCAM. ${ }^{* *} \mathrm{P}<0.01$ vs. NC group; ${ }^{\# \prime} \mathrm{P}<0.01$ vs. AS group; \&\& $<<0.01$ as indicated; AS, atherosclerosis; NC, normal control; si, small interfering; HOXA9, homeobox A9; PF4, platelet factor-4; VCAM-1, vascular cell adhesion molecule-1.

alleviate symptoms of AS via relieving vascular injury and lipid formation.

Expression of HOXA9 increases in coronary arteries of AS rats. Immunohistochemical analysis was initially conducted to examine the protein expression levels of HOXA9 in the coronary arteries of rats. Higher levels of HOXA9 protein, localized mainly in the wall of coronary arteries (depicted by black arrows), were observed in the AS model rats when compared with that in the NC group (Fig. 3C). The treatment of rats with HOXA9 inhibitors or si-HOXA9 reduced the levels of HOXA9 protein compared with the AS group (Fig. 3C). Taken together, the qualitative expression of HOXA9 detected by immunohistochemistry presented a preliminary verification of the positive association between HOXA9 levels and coronary arteries morphology of AS rats.

HOXA9 inhibitors alleviate AS damage by downregulating PF4 and E-selectin/VCAM-1 proteins. To investigate the mechanisms by which HOXA9 inhibitors alleviate AS symptoms, western blotting was employed to detect the protein expression levels of HOXA9, PF4, E-selectin and VCAM-1. The results revealed that the expression levels of HOXA9, PF4, E-selectin and VCAM-1 were significantly upregulated in the AS group compared with those in the NC group (Fig. 4). As shown in Fig. 4, the injection of HOXA9 inhibitors or 
si-HOXA9 markedly decreased the expression levels of these proteins. Overexpression of PF4 in the HOXA9 inhibitor group significantly upregulated the expression of PF4, E-selectin and VCAM-1. E-selectin overexpression resulted in no effects on the levels of PF4, but increased the levels of E-selectin and VCAM-1. Moreover, VCAM-1 overexpression upregulated VCAM-1, but had no effects on PF4 and E-selectin. Therefore, these findings suggested that HOXA9 inhibitors may alleviate AS damage through reducing the levels of PF4, and subsequently downregulating E-selectin and VCAM-1 (Fig. 4F).

\section{Discussion}

AS is a cardiovascular disease that results in high morbidity and mortality rates. Therefore, it is necessary to study the underlying pathological mechanisms in order to identify potential treatments (21). The progression of AS could be regarded as a chronic inflammatory disease in arterial walls, where the expression levels of pro-inflammatory cytokines and adhesive factors are upregulated. A previous study demonstrated that microRNA-27 suppressed the lipid accumulation and inflammatory response in apolipoprotein E-knockout mice, suggesting that the inflammatory status in coronary arteries could be regarded as an indicator of the progression of AS (22).

HOXA9, a member of the HOX gene family, participates in the differentiation of promyelocytic cells in the hematopoietic system and is involved in cellular processes such as proliferation, differentiation and apoptosis of ECs (23). It was reported that HOXA9-knockout mice were deficient in the production of mature granulocytes and B lymphocytes, and exhibited impaired T-cell differentiation with increased apoptosis (23). To investigate whether HOXA9 expression affects the development of AS, the present study successfully constructed AS rat models through oral feeding of a high-fat and high-cholesterol diet along with an intravenous injection of vitamin D3. The results demonstrated that inhibiting HOXA9 decreased the levels of inflammatory cytokines, including TNF- $\alpha$, IL-1 $\beta$ and IL-12. Consistent with the present study, Trivedi et al (24) demonstrated that HOXA9 participated in maintaining the inactivated state of ECs and inhibited the expression of adhesive factors induced by TNF- $\alpha$ through inhibiting nuclear factor (NF) $-\kappa B$.

The present study also demonstrated that the levels of CCL25 were downregulated following inhibition of HOXA9. As a major mediator in macrophage chemotaxis, CCL25 has been shown to specifically induce M1 macrophage chemotaxis, thus leading to the further progression of AS (25). Consistently, in the present study, the content of M1 macrophages was increased in AS rats compared with the NC group, whereas treatment with HOXA9 inhibitors resulted in a decrease in M1 macrophages. The observation of the morphology of coronary arteries using H\&E staining further supported these findings, suggesting that HOXA9 inhibition alleviated the AS symptoms and improved the blood microcirculation. In order to observe the morphology of coronary arteries, a previous study implemented the use of a Leica image analysis system. The size of plaques in coronary arteries of AS rats was found to be significantly diminished using onion extracts to suppress vascular inflammation (26).

The results of western blotting elucidated that HOXA9 inhibitors downregulated the expression levels of PF4 and adhesion factors E-selectin and VCAM-1. PF4 has been reported to be upregulated in AS, and to participate in the accumulation of monocytes and monocyte-derived cells, which can further express inflammatory cytokines and induce expression of adhesion factors (27). It has been demonstrated that irbesartan attenuated TNF- $\alpha$-induced expression of E-selectin, ICAM-1 and VCAM-1 via suppression of the NF- $\mathrm{NB}$ pathway in human umbilical vein endothelial cells (28). A previous study found that treatment with $\beta$-thujaplicin counteracted AS symptoms by decreasing E-selectin and VCAM-1 (29). These findings suggested that HOXA9 inhibitors may relieve the progression of AS via suppression of PF4 and E-selectin/VCAM-1 protein expression, supporting the potential use of HOXA9 inhibitors in the therapy of AS.

In the present study, HOXA9 inhibition resulted in both a decrease in the inflammatory status and an improvement in the microcirculation of coronary arteries in AS rats, via downregulation of PF4, E-selectin and VCAM-1 protein expression. Thus, the inhibition of HOXA9 may have potential as a treatment option for AS; however, further studies are needed to confirm this.

\section{Acknowledgements}

Not applicable.

\section{Funding}

No funding was received.

\section{Availability of data and materials}

The datasets used and/or analyzed during the current study are available from the corresponding author on reasonable request.

\section{Authors' contributions}

SL, JG and SW conducted experiments, analyzed data and assisted with the drafting or revision of the manuscript. SL and JG confirm the authenticity of the raw data. All authors have read and approved the final version of the manuscript.

\section{Ethics approval and consent to participate}

The present study was approved by the Medical Ethics Committee of Shengli Oilfield Central Hospital (approval no. 2019051).

\section{Patient consent for publication}

Not applicable.

\section{Competing interests}

The authors declare that they have no competing interests. 


\section{References}

1. Libby P, Lichtman AH and Hansson GK: Immune effector mechanisms implicated in atherosclerosis: From mice to humans. Immunity 38: 1092-1104, 2013.

2. Kwon GP, Schroeder JL, Amar MJ, Remaley AT and Balaban RS Contribution of macromolecular structure to the retention of low-density lipoprotein at arterial branch points. Circulation 117: 2919-2927, 2008.

3. Bäck M and Hansson GK: Anti-inflammatory therapies for atherosclerosis. Nat Rev Cardiol 12: 199-211, 2015.

4. Herrero-Fernandez B, Gomez-Bris R, Somovilla-Crespo B and Gonzalez-Granado JM: Immunobiology of atherosclerosis: A complex net of interactions. Int J Mol Sci 20: 5293, 2019.

5. Gimbrone MA Jr and García-Cardeña G: Endothelial cell dysfunction and the pathobiology of atherosclerosis. Circ Res 118: 620-636, 2016.

6. Barrett TJ: Macrophages in atherosclerosis regression. Arterioscler Thromb Vasc Biol 40: 20-33, 2020.

7. Chistiakov DA, Orekhov AN and Bobryshev YV: Endothelial barrier and its abnormalities in cardiovascular disease. Front Physiol 6: 365, 2015.

8. Chistiakov DA, Melnichenko AA, Myasoedova VA, Grechko AV and Orekhov AN: Mechanisms of foam cell formation in atherosclerosis. J Mol Med (Berl) 95: 1153-1165, 2017.

9. Bakogiannis C, Sachse M, Stamatelopoulos K and Stellos K Platelet-derived chemokines in inflammation and atherosclerosis. Cytokine 122: 154157, 2019.

10. Iademarco MF, Barks JL and Dean DC: Regulation of vascular cell adhesion molecule- 1 expression by IL- 4 and TNF-alpha in cultured endothelial cells. J Clin Invest 95: 264-271, 1995.

11. Cantile M, Schiavo G, Terracciano L and Cillo C: Homeobox genes in normal and abnormal vasculogenesis. Nutr Metab Cardiovasc Dis 18: 651-658, 2008.

12. Duboule D and Morata G: Colinearity and functional hierarchy among genes of the homeotic complexes. Trends Genet 10: 358-364, 1994

13. Gorski DH and Walsh K: The role of homeobox genes in vascular remodeling and angiogenesis. Circ Res 87: 865-872, 2000.

14. Bandyopadhyay S, Ashraf MZ, Daher P, Howe PH and DiCorleto PE: HOXA9 participates in the transcriptional activation of E-selectin in endothelial cells. Mol Cell Biol 27: 4207-4216, 2007.

15. Pang J, Xu Q, Xu X, Yin H, Xu R, Guo S, Hao W, Wang L, Chen C and Cao JM: Hexarelin suppresses high lipid diet and vitamin D3-induced atherosclerosis in the rat. Peptides 31: 630-638, 2010.

16. Zheng $\mathrm{X}$ and $\mathrm{Hu} \mathrm{X}$ : Effects of rosuvastatin on hypertension with carotid atherosclerosis and its influence on peripheral macrophage polarization. Chinese Journal of Clinical Pharmacology and Therapeutics 22: 1035-1039, 2017.

17. Wang J, Hou J, Lin C, Fu J, Ren J, Li L, Guo H, Han X, Wang B and Liu J: Shuangshen ningxin capsule, a traditional Chinese medicinal preparation, alleviates myocardial ischemia through autophagy regulation. Evid Based Complement Alternat Med 2015: 581260, 2015.
18. Xu B, Geerts D, Bu Z, Ai J, Jin L, Li Y, Zhang H and Zhu G: Regulation of endometrial receptivity by the highly expressed HOXA9, HOXA11 and HOXD10 HOX-class homeobox genes. Hum Reprod 29: 781-790, 2014

19. Sun X, He S, Wara AKM, Icli B, Shvartz E, Tesmenitsky Y, Belkin N, Li D, Blackwell TS, Sukhova GK, et al: Systemic delivery of microRNA-181b inhibits nuclear factor- $\kappa$ B activation, vascular inflammation, and atherosclerosis in apolipoprotein E-deficient mice. Circ Res 114: 32-40, 2014.

20. Shirai T, Hilhorst M, Harrison DG, Goronzy JJ and Weyand CM: Macrophages in vascular inflammation-From atherosclerosis to vasculitis. Autoimmunity 48: 139-151, 2015.

21. Nitsa A, Toutouza M, Machairas N, Mariolis A, Philippou A and Koutsilieris M: Vitamin D in cardiovascular disease. In Vivo 32: 977-981, 2018.

22. Xie W, Li L, Zhang M, Cheng HP, Gong D, Lv YC, Yao F, He PP, Ouyang XP, Lan G, et al: MicroRNA-27 prevents atherosclerosis by suppressing lipoprotein lipase-induced lipid accumulation and inflammatory response in apolipoprotein E knockout mice. PLoS One 11: e0157085, 2016.

23. Calvo KR, Sykes DB, Pasillas M and Kamps MP: Hoxa9 immortalizes a granulocyte-macrophage colony-stimulating factor-dependent promyelocyte capable of biphenotypic differentiation to neutrophils or macrophages, independent of enforced meis expression. Mol Cell Biol 20: 3274-3285, 2000.

24. Trivedi CM, Patel RC and Patel CV: Homeobox gene HOXA9 inhibits nuclear factor-kappa B dependent activation of endothelium. Atherosclerosis 195: e50-e60, 2007.

25. Xuan W, Qu Q, Zheng B, Xiong S and Fan GH: The chemotaxis of M1 and M2 macrophages is regulated by different chemokines. J Leukoc Biol 97: 61-69, 2015.

26. Li W, Tang C, Jin H and Du J: Effects of onion extract on endogenous vascular $\mathrm{H} 2 \mathrm{~S}$ and adrenomedulin in rat atherosclerosis. Curr Pharm Biotechnol 12: 1427-1439, 2011.

27. Domschke G and Gleissner CA: CXCL4-induced macrophages in human atherosclerosis. Cytokine 122: 154141, 2019.

28. Jiang Y, Jiang LL, Maimaitirexiati XM, Zhang Y and Wu L: Irbesartan attenuates TNF- $\alpha$-induced ICAM-1, VCAM-1, and E-selectin expression through suppression of NF- $\kappa \mathrm{B}$ pathway in HUVECs. Eur Rev Med Pharmacol Sci 19: 3295-3302, 2015.

29. Shih MF, Pan KH, Liu CC, Shen CR and Cherng JY: Treatment of $\beta$-thujaplicin counteracts di(2-ethylhexyl)phthalate (DEHP)-exposed vascular smooth muscle activation, inflammation and atherosclerosis progression. Regul Toxicol Pharmacol 92: 333-337, 2018.

This work is licensed under a Creative Commons Attribution 4.0 International (CC BY-NC 4.0) License 